\title{
Activation of hepatic stellate cell in Pten null liver injury model
}

\author{
Lina He', James Gubbins², Zhechu Peng ${ }^{1}$, Vivian Medina', Fan Fei', Kinji Asahina ${ }^{3}$, Jiaohong Wang ${ }^{3}$, \\ Michael Kahn ${ }^{1,4}$, Carl B. Rountree ${ }^{5}$ and Bangyan L. Stiles ${ }^{1,3,6^{*}}$
}

\begin{abstract}
Background: Hepatic fibrosis is a prominent pathological feature associated with chronic liver disease including non-alcoholic hepatosteatosis (NASH), and a precursor for liver cancer development. We previously reported that PTEN loss in the liver, which leads to hyperactivated liver insulin signaling results in NASH development. Here we used the same mouse model to study the progression from steatosis to fibrosis.

Results: The Pten null livers develop progressive liver fibrosis as indicated by Sirius Red staining and increased expression of collagen I, Timp 1, SMAa, and p75NTR. Consistently, hepatic stellate cells (HSCs) isolated from Pten null livers are readily activated when compared with that from mice with intact PTEN. Deletion of AKT2, the downstream target of PTEN signal, blocked NASH development, and alleviated fibrosis. HSCs from the Pten/Akt2 double null mice are quiescent like those isolated from the control livers. Our analysis shows that the activation of HSCs does not depend on the intrinsic signals regulated by PI3K/AKT, the target of PTEN, but does depend on steatosis and injury to the liver. During the progression of liver fibrosis in the Pten null model, Wnt ligands and signaling receptor are induced, concurrent with the reduction of SFRP5, a Wnt antagonist. We showed that treatment of HSCs with Wnt receptor antagonist blocks the observed morphological changes when HSCs undergo activation in culture. This signal appears to be mediated by $\beta$-catenin, as manipulating $\beta$-catenin signaling alters marker gene expressions of HSC activation.
\end{abstract}

Conclusions: Wnt/ $\beta$-catenin activation serves as an important mediator for fibrosis development resulting from NASH using a mouse model where NASH is mimicked by PTEN loss.

Keywords: Fibrosis, Wnt, PTEN, AKT, Fatty liver, Steatosis

\section{Background}

Fatty liver disease (FLD) is the most prevalent form of chronic liver disease and has become a major pandemic in developed and some developing countries. FLD is characterized by lipid accumulation in the liver resulting from sedentary lifestyle, calorie-rich diet, or alcohol consumption. Without intervention, FLD can develop into non-alcoholic or alcoholic hepatosteatosis (NASH or ASH) where fatty liver is accompanied by infiltration of inflammatory cells. Left untreated, NASH and ASH can

\footnotetext{
*Correspondence: bstiles@usc.edu

'Pharmacology and Pharmaceutical Sciences, School of Pharmacy, University

of Southern California, Los Angeles, CA 90033, USA

${ }^{3}$ Pathology, Keck School of Medicine, University of Southern California, Los

Angeles, CA 90033, USA

Full list of author information is available at the end of the article
}

develop into fibrosis, a common late stage chronic liver disease and a significant risk factor for cancer [1].

The mechanisms responsible for this fibrosis development in NASH/ASH patients are not well studied but may involve injury-repair responses and accumulation of myofibroblasts. Myofibroblasts are thought to be the cells that lay down the collagens that compose the fibrotic tissue [2]. Of the potential sources for these myofibroblasts, activation of hepatic stellate cells (HSCs) has gained strong support [3]. Inactive HSCs are the main storage for vitamin A. Upon injury, HSCs activate by losing their vitamin A containing lipid droplets and gaining myofibroblast characteristics. This morphological alteration is associated with increased expressions of markers such as, collagen type I (Colla1), smooth muscle actin $\alpha(\mathrm{SMA} \alpha)$, desmin, and nerve growth factor receptor (P75NTR). Multiple stimuli and pathways have 
been shown to stimulate the expression of these markers in HSCs. The most prominent among these are transforming growth factor $\beta$ (TGF $\beta$ ) and platelet-derived growth factor (PDGF) [4].

To investigate the molecular signals involved in fibrosis resulting from fatty liver, we used a murine model where steatosis is induced as a result of hyperactivated insulin signal, a condition that commonly occurs in NASH patients. In this mouse model (hereafter referred to as Pten null mice), Pten (phosphatase and tensin homologue deleted on chromosome 10) is deleted in the albuminpositive cell population (Pten $\left.{ }^{\text {loxP/loxP }} ; \mathrm{Alb}-\mathrm{Cre}^{+}\right)$. In such mice, the liver PI3K/AKT pathway responsible for transmitting insulin signal is induced without the complication of peripheral insulin resistance and hyperglycemia [5]. The Pten null mouse model thus mimics the liver signals occurring in NASH patients where hyperinsulinemia often drives lipid synthesis in the liver. In this model, we have previously observed significant injury to the liver and ultimately liver tumor development [5-7]. In the current study, we characterize the fibrosis development in Pten null mice and validated it as an appropriate model to study the contribution of NASH to fibrosis. In addition, we investigated the mechanisms underlying the steatosis contribution to fibrosis development using this model.

\section{Results}

PTEN levels are lower in NASH patients

Liver fibrosis/cirrhosis often accompanies the development of fatty liver as confirmed here with Sirius Red staining (Fig. 1a). Using a published data set (GSE37031) [8], we found that expression of PTEN, a lipid phosphatase is negatively correlated with the presence of NASH (Fig. 1b). The protein expression of PTEN is further verified by analyzing

A

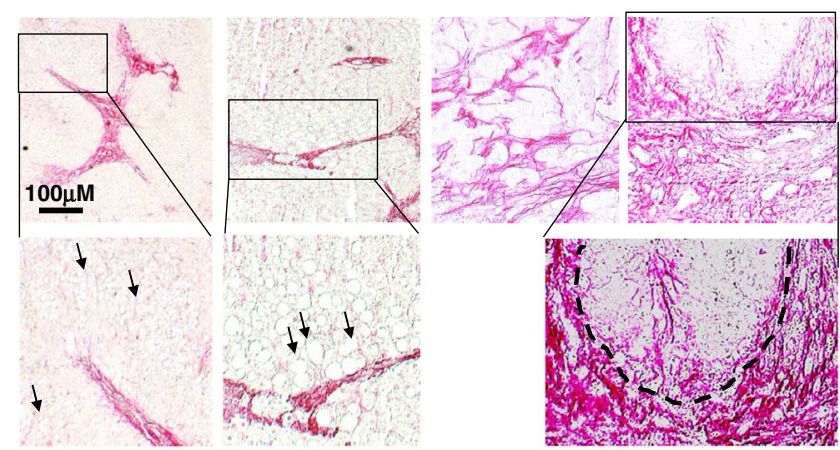

$\mathrm{B}$
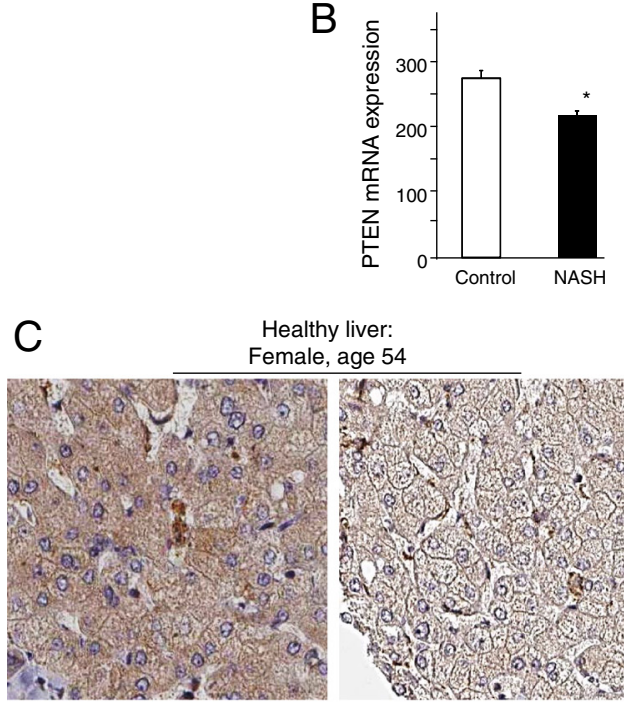

Healthy liver:
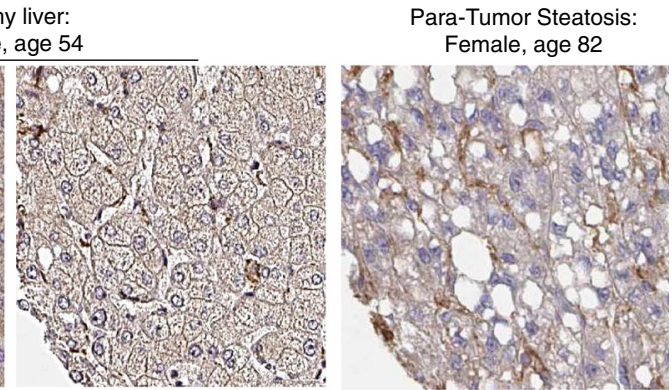

Fig. 1 Association of fibrosis and PTEN status with NASH. a Sirius Red staining for fibrosis in biopsied liver human non-alcoholic steatohepatitis (NASH) patients. Biopsy livers were obtained from patients with various degrees of diagnosed NASH. Sirius Red staining is performed on the livers to identify fibrotic regions. Top two panels, fibrosis near fatty liver deposits; bottom up second panel, premalignant lesions with intensive Sirius Red staining; bottom panel, areas with fibrotic tissues only. Right panels, higher magnified images of the cropped areas from the left panels. Arrows: lipid deposit in hepatocytes. Dotted enclosure, premalignant lesion. b PTEN expression is lower in NASH vs. control samples. Publically available data set (GSE37031) was analyzed for expression of PTEN. ${ }^{*} p<0.05$. $\mathbf{c}$ Immunohistochemical analysis of PTEN protein in two patients. Images generated by The Human Protein Atlas (www.proteinatlas.org) 
images obtained from the Human Protein Atlas (http:// www.proteinatlas.org). Within healthy liver, PTEN expression is lower in hepatocytes with micro-vesicular lipid droplets (Fig 1, left two panels). In steatotic liver tissues from HCC patient, PTEN expression is only detected in non-hepatocytes (Fig. 1c).

\section{Pten null mice develop progressive liver fibrosis}

In mouse models, PTEN loss in the liver leads to hyperactivation of the PI3K/AKT pathway, leading to progressive NASH followed by spontaneous tumor development [5-7]. Consistent with our previous results in young mice [5], the older Pten null mice in the current study displayed lower body weight and plasma glucose (Fig. 2a, b) throughout all age cohorts. At 6 months of age, all mice exhibit fatty liver disease with adenomas and hyperproliferation of the ductal epithelial similar to Von Meyenburg syndrome (Fig. 2c). Pericellular staining of Sirius Red is observed in areas of steatosis at this age (Fig. 3a) and becomes progressively more severe in 9- and 12-month-old Pten null mice (Fig. 3b, c), consistent with clinical observations where fibrosis accompanies steatosis. In addition to Pten null mice, we also evaluated Sirius Red in a model in which both Pten and $A k t 2$ are deleted. The deletion of $A k t 2$ eliminates the occurrence of NASH that develops in mice lacking PTEN alone [7]. Consistent with the lack of NASH status of the Pten/Akt2 double-deleted mice, only isolated ducts are stained with Sirius Red in the Pten/Akt2 double null mice, similar to the control livers (Fig. 3a). Together, our data suggests that PTEN loss leads to fibrosis development and AKT2 plays a role in this development.
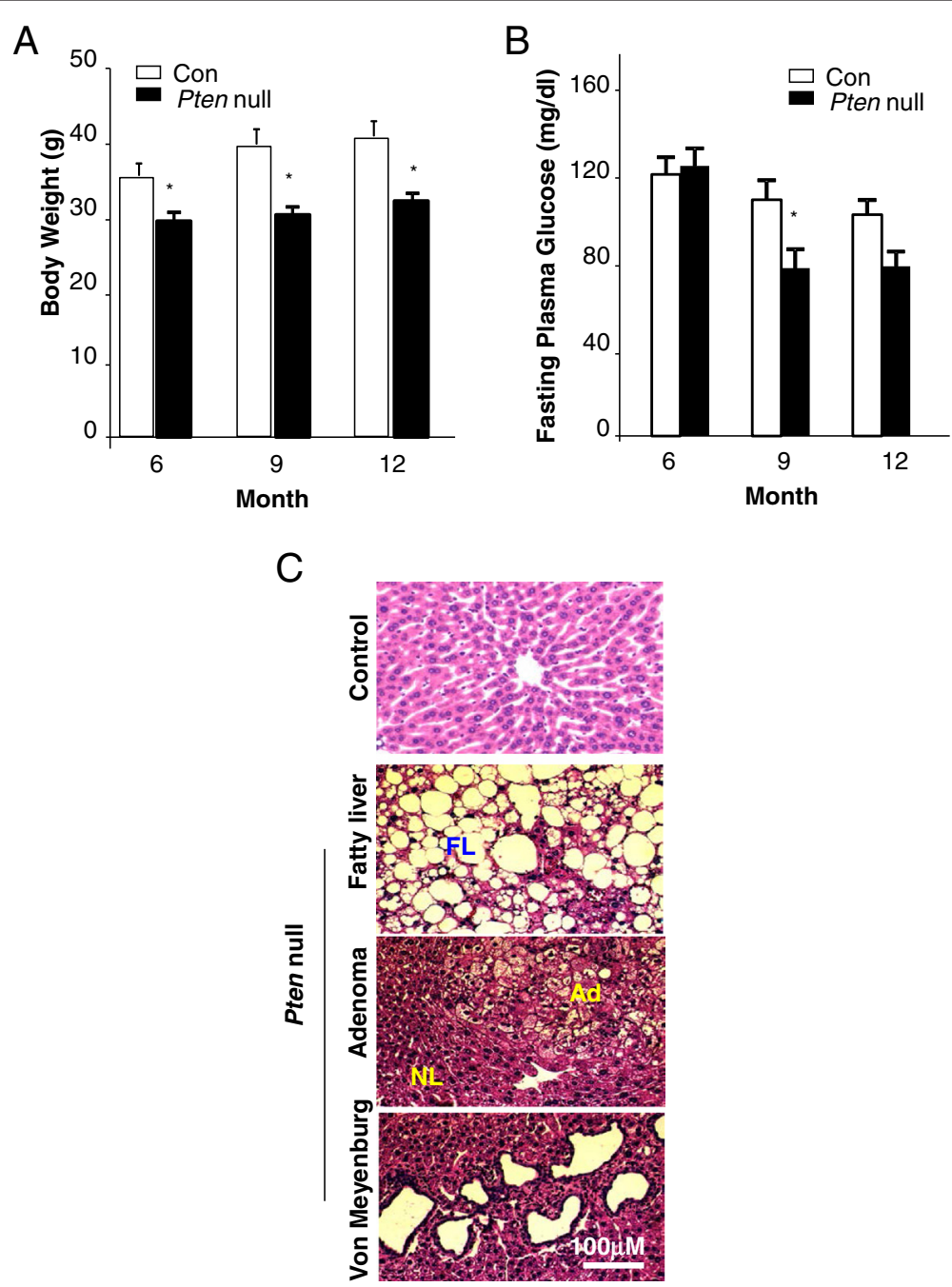

Fig. 2 Phenotypes of mice carrying the deletion of Pten (Pten null) in the liver. a Body weight of control and Pten null mice. b Fasting plasma glucose in control and Pten null mice. $n=7-10 .{ }^{*} p<0.05$. c Liver-specific Pten deletion mice develop fatty liver disease at an early age (top; FL fatty liver). Adenomas are also observed in these mice (middle, $n /$ normal liver, $A d$ adenomas). Morphologies that resemble Von Meyenburg syndrome are also observed in the livers of the liver Pten null mice (bottom) 

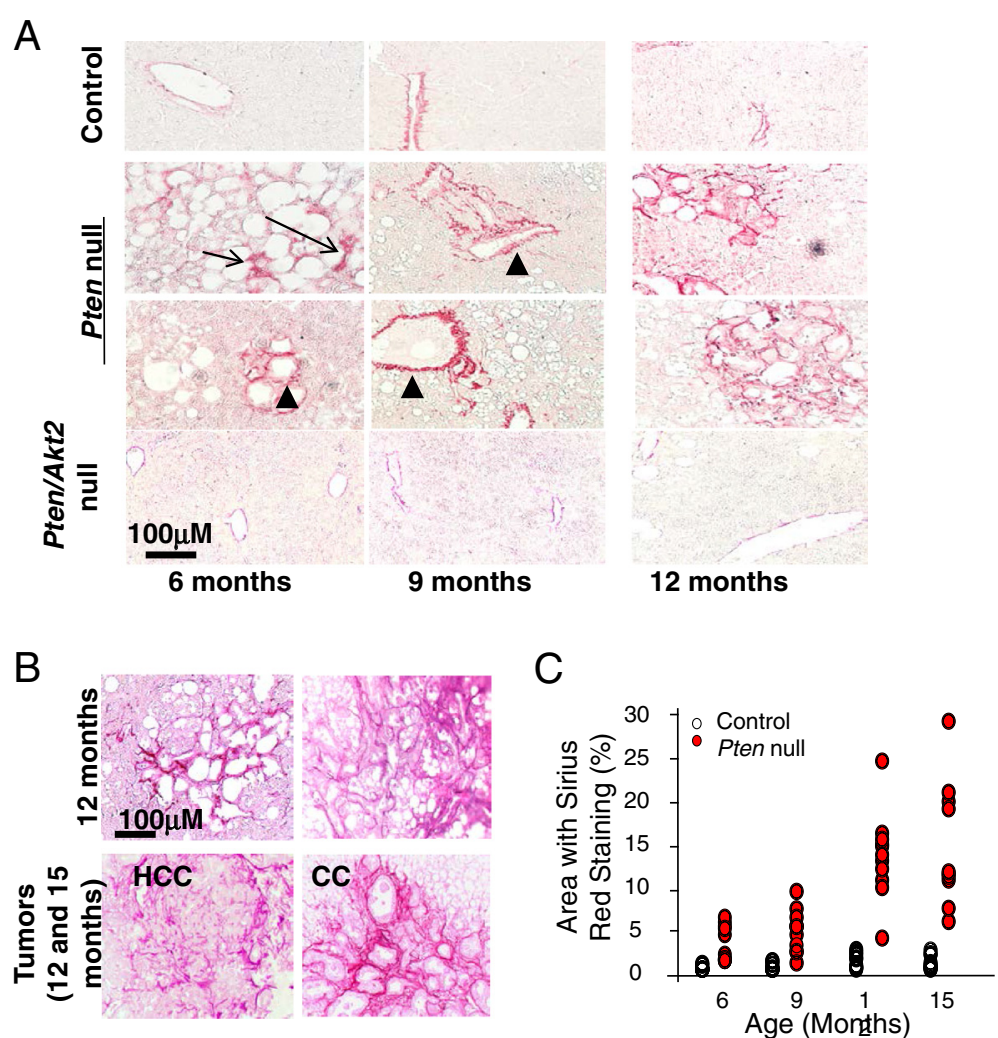

Fig. 3 Pten null livers develop progressive fibrosis that is attenuated by AKT2 loss. a Periductal (arrowhead) and pericellular (arrows) staining of Sirius Red are obvious in the 6- and 9-month-old Pten null livers, indicating fibrosis development. In older mice (12 months of age), more intensive Sirius Red staining is observed. In Pten and Akt2 double mutants (Pten/Akt2 null), minimum staining for Sirius Red is observed (bottom row). Top row, controls; middle two rows, Pten null livers. b Sirius Red staining in tumor samples from the Pten null mice. c Sirius Red stained areas were quantified vs. non-stained areas. Percentage of total area that is positive for Sirius Red is reported. Each circle represents an animal. Open circle, control; solid red circle, Pten null

To further confirm the fibrosis pathology, we analyzed the expression of several fibrosis markers: Col1a1, desmin, SMA $\alpha$, and p75NTR as well as tissue inhibitor of metalloproteases 1 (Timp 1) to quantitatively assess the buildup of collagen fibers. We show that expressions of these markers are significantly upregulated in 9- and 12month-old Pten null livers (Fig. 4a). Consistent with a role of AKT2 in the development of fibrosis, the expression of these fibrogenic genes were reduced when Akt2 is deleted simultaneously with Pten. The only exception is Timp 1 expression in 9-month-old mice. The expression of Timp 1 increased by two- to threefolds in Akt2/ Pten double mutants vs. Pten deletion alone. The lack of downregulation in Timp 1 by Akt2 deletion and its decline in 12- vs. 9-month-old mice may be related to its potential role in tumorigenesis as all Pten null mice developed tumors at 12 months of age and Timp 1 has been shown to inhibit mitogenesis in HCC [9].

To confirm these gene expression changes, we stained liver sections for SMA . In 9-month-old Pten null mice, this staining is mostly observed in areas of severe fatty liver and the ductal plates (Fig. 4b). In 12-month-old mice, SMA $\alpha$ staining is distributed throughout the Pten null livers, whereas the Pten/Akt2 double null and control livers have little staining for SMA $\alpha$ at either age. The SMA $\alpha$ staining is further confirmed with immunoblotting analysis showing increased SMA $\alpha$ in 9- and 12month-old mice (Fig. 4c). These observations show that Pten deletion leads to progressive and severe fibrosis that is recovered when AKT2 is simultaneously lost.

\section{HSCs are activated in Pten null liver}

The buildup of collagen fibers and upregulation of Colla1 and Timp 1 have been attributed to the activation of HSCs. We isolated HSCs from the 9-month-old Pten null (HSC-Pm) and control livers (HSC-Con). The majority of the HSC-Con cells displayed rounded shapes and intense vitamin A autofluorescence, indicating quiescent HSCs (Fig. 5a). HSC-Pm was a mixture of rounded quiescent HSCs and cells with elongated myofibroblast morphologies (Fig. 5a). Expressional analysis of markers indicates that Colla1 $\left(5.4 \times 10^{-2}\right.$ vs. $\left.5.9 \times 10^{-3}\right)$ and SMA $\alpha\left(1.9 \times 10^{-3}\right.$ vs. $\left.2.2 \times 10^{-4}\right)$ are more than fivefold higher in HSC-Pten null cells when compared with 

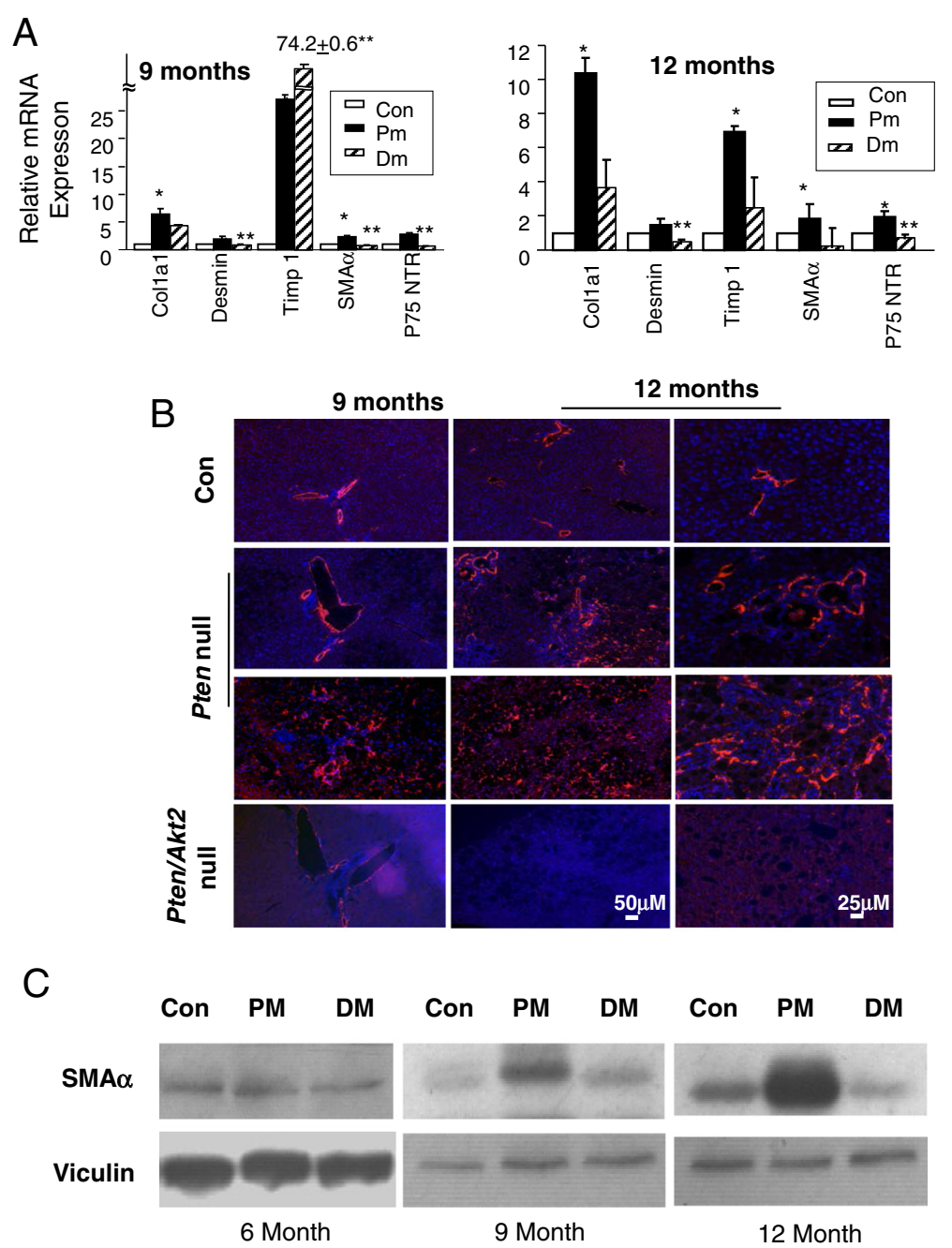

Fig. 4 Fibrosis markers are upregulated in the Pten null livers and reduced when Akt2 is simultaneously deleted. a Expression analysis of markers for fibrosis, i.e., desmin, SMAa, Timp 1, Col1a1, and p75NTR are analyzed in 9 (left)- and 12 (right)-month-old Pten control (Con) and null livers (Pm) as well as the Pten/Akt2 double null livers (Dm). Solid bars, Pten null liver; stripped bars, Pten/Akt2 double null; open bars, control liver. Striped bars, Pten/Akt2 double null. *significantly different from controls of the same gene analyzed; **significantly different from Pten null group of the same gene analyzed. $n=5 . p<0.05$. Three animals each group are used for the experiment with each sample repeated twice. $\mathbf{b}$ Liver tissues were stained with SMAa antibody using indirect immunofluorescence staining (red). The same slides were counterstained with DAPI (blue) to visualize nucleus. Left panels, 9-month-old livers; middle panels, 12-month-old livers; right panels, high mag images of 12-month-old livers. Top panels, control; middle two rows, Pten null; bottom row, Pten and Akt2 double mutant (Pten/Akt2 null). $\mathbf{c}$ Immunoblotting analysis of SMAa in liver lysate isolated from the indicated mouse models. Pten control (Con) and null livers (Pm) as well as the Pten/Akt2 double null livers (Dm)

HSC-Con (Fig. 5b). Timp 1 expression is also threefold higher in HSC-Pm vs. HSC-Con. Expression of these markers is significantly reduced in HSC isolated from the Pten/Akt2 double null mice (HSC-Dm) compared to their expression in HSC-Pm cells (Fig. 5c). No difference in p75NTR and desmin expression was observed in the freshly isolated HSCs (data not shown).

The morphological differences between the two HSC cultures are more obvious after the attachment of HSCs at day 2 of culturing (Fig. 5d). The majority of the HSCCon cells are still round-shaped quiescent HSCs retaining vitamin A autofluorescence, whereas most HSC-Pm cells acquired spindle morphology with very little vitamin A deposits, indicating that they are activated HSCs. HSC-Dm displayed similar quiescent phenotype and retained vitamin A autoflorescence. Like reported, HSCCon gradually changed from the round-shaped quiescent HSC phenotype to the long, stretched, spindle phenotype that resembles activated HSC from days 3 to 7 (data not shown). By day 5, majority of the HSC-Con are activated and by day 7 , all cells display the elongated spindle morphology. HSCs from Pten null mice changed very little morphologically as they already acquired the spindle morphology by day 2 . The cells appear to shrink with 


\section{A}
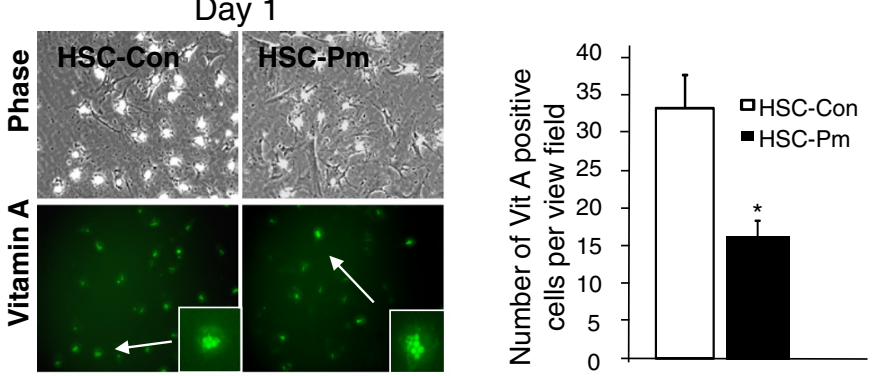

B
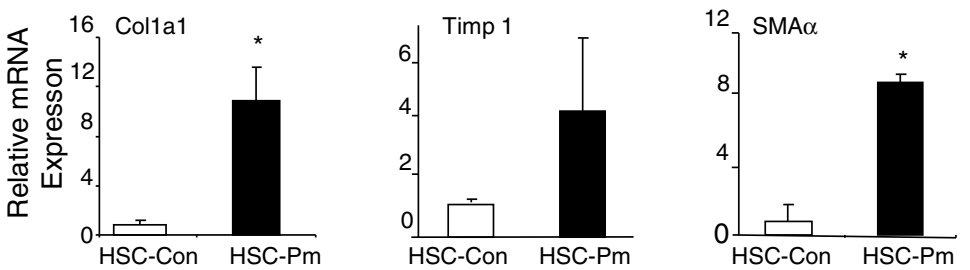

C

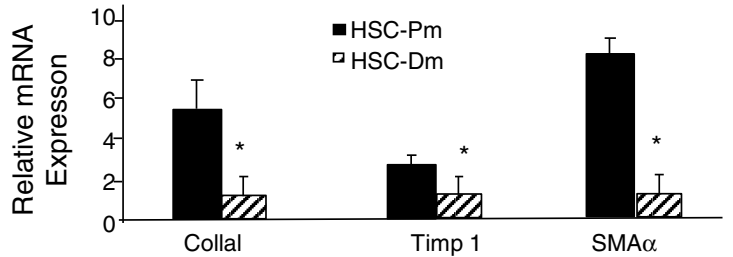

D
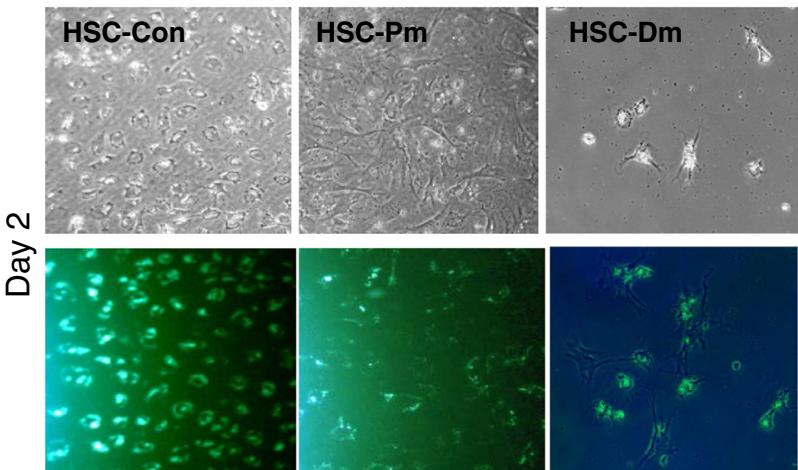

Fig. 5 Hepatic stellate cells (HSCs) from the Pten null mice are activated. a Morphology of HSCs isolated from Pten control (HSC-Con) and null (HSC-Pm) mice after overnight culturing for attachment. Top left panel, phase contrast images; bottom left panels, autofluorescence of vitamin A. Green fluorescence (pseudo-colored green) indicates vitamin A-rich oil droplets. Inset, higher magnification images of the oil droplets with vitamin A autofluorescence. Right panel, quantification of vitamin A-positive cells. ${ }^{*}$ significantly different between the two groups at $p<0.05$. At least three randomly selected views per culture were used for measurement. $\mathbf{b}$ Expression of fibrosis markers (Col1a1, SMAa, and Timp 1) indicates that HSCs from Pten null livers are activated. Open bar, HSCs isolated from control livers (HSC-Con); solid bar, HSCs isolated from Pten null livers (HSC-Pm). * significantly different between the two groups at $p<0.05 . n=3$. c Expression of fibrosis markers in HSC isolated from the Pten null (HSC-Pm) and Pten/ Akt2 double null (HSC-Dm) livers. * significantly different between the two groups at $p<0.05 . n=3$. d HSCs from Pten null livers are fully activated at day 2 of culture where HSCs from control (HSC-Con) and Pten/Akt2 double null (HSC-Dm) livers still remain quiescent. Top panel, phase contrast images; bottom panel, autofluorescence of vitamin A (pseudo-colored green)

time, likely due to increasing cell density with culturing (data not shown). Consistent with the morphological changes occurring in HSC-Con culture, expressional analysis of Col1a1, Timp 1, and SMA $\alpha$ increased with culturing (data not shown).
Activation of HSC in Pten null mice depends on injury but not intrinsic PI3K/AKT activation

Constitutively activation of AKT results in induction of collagen I [10] whereas the introduction of dominant negative PI3K inhibits Timp 1 and SMA $\alpha$ [11]. We 
evaluated whether PI3K/AKT signal may be chronically activated in HSC-Pm isolated from the Pten null mice and could be responsible for the phenotypes. While the levels of PTEN decreased moderately in the HSC-Pm, the levels of p-AKT did not differ significantly between HSC-Pm and HSC-Con cells (Fig. 6a). Furthermore, levels of p-GSK3 $\beta$, a substrate of AKT did not increase either. Instead, p-GSK3 $\beta$ and GSK3 $\beta$ levels are slightly lower in the HSCs from Pten null mice. This observation suggests that intrinsic signals due to PI3K activation are unlikely to play a role in the activated HSC observed in the Pten null liver. Thus, HSC activation phenotype in Pten null mice is likely independent of the intrinsic PI3K signaling pathways.

To further confirm this observation, we treated rat HSC cell line with insulin growth factor (IGF-1) to induce PI3K/AKT. In these cells, overnight starvation led to moderate downregulation of p-AKT. When IGF-1 is added, AKT phosphorylation is induced both 15 min and $1 \mathrm{~h}$ after the start of the treatment (Fig. 6b). Phosphorylation of AKT starts to recover after $1 \mathrm{~h}$ (data not shown). We evaluated the expression levels of SMA $\alpha$, Colla1, Timp 1, and desmin (Fig. 6c). The expression of most fibrotic genes did not alter significantly when p-AKT is induced in the IGF-1
A

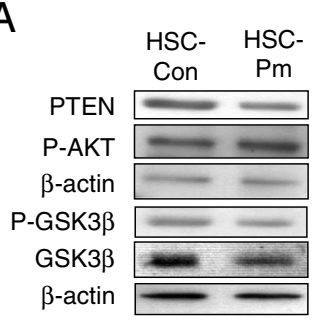

B

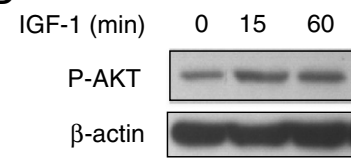

D

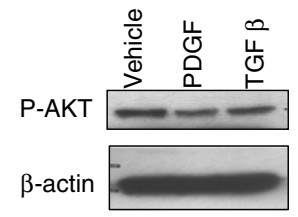

$\mathrm{F}$

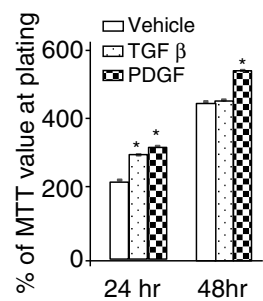

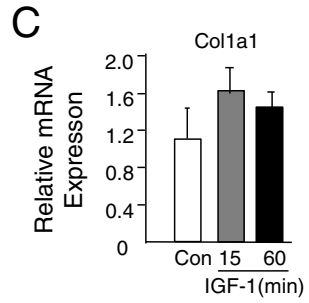
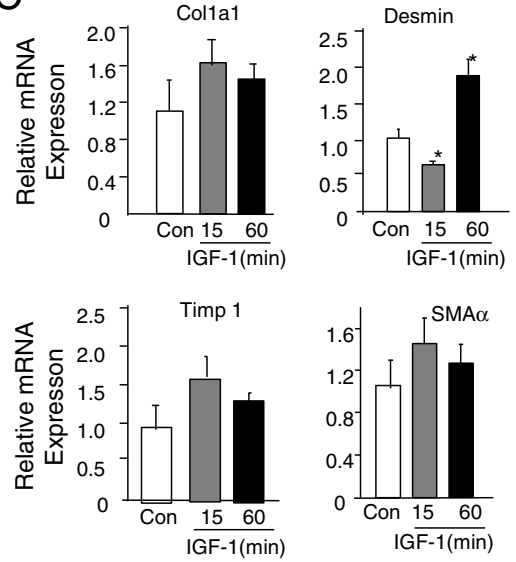

$E$

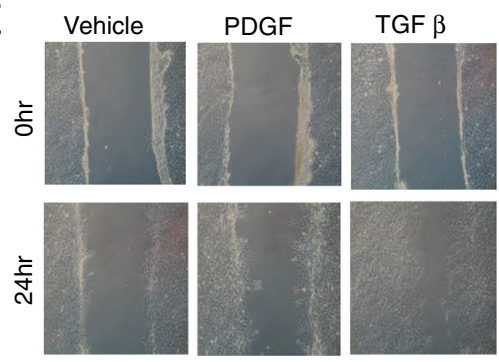

G

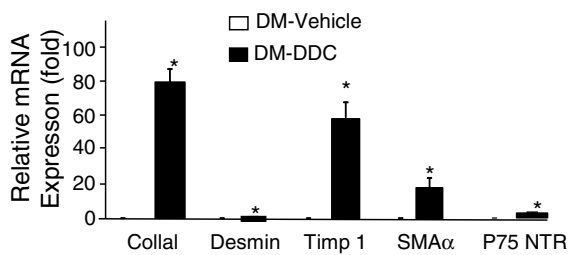

Fig. 6 PTEN regulates HSC gene expression independent of AKT signal. a. Protein analysis of HSCs isolated from control (HSC-Con) and Pten null (HSC-Pten

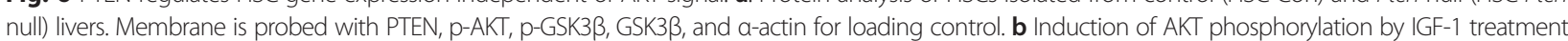
in human HSC cell line. $\beta$-actin is used as loading control. c Quantitative PCR analysis of expression of SMAa, Col1al, Timp 1, and desmin in vehicle- and IGF-1 (gray bar, 15 min; and black bar, 1 h)-treated samples, respectively. $n=3,{ }^{*} p<0.05$ when compared to the vehicle-treated group. $\mathbf{d}$ Analysis of AKT phosphorylation in response to PDGF $(50 \mathrm{ng} / \mathrm{ml})$ and TGF $\beta(4 \mathrm{ng} / \mathrm{ml})$ treatment. e PDGF and TGF $\beta$ treatment induced HSC migration as measured with a wound healing assay. Representative images of three experiments. $\mathbf{f}$ PDGF and TGF $\beta$ both induced cell proliferation in cultured HSCs. Cell proliferation is measured by a MTT assay. $n=3,{ }^{*} p<0.05$. g Relative expression of SMAa, Col1al, Timp 1, p75NTR, and desmin in livers of Pten and Akt2 double mutant (Dm) mice treated with (black bar) or without DDC (open bar). $n=5,{ }^{*} p<0.05$ when compared to the vehicle-treated group 
treated HSCs with the exception of desmin expression. At $15 \mathrm{~min}$, we observed a significant downregulation of desmin whereas this level increased after $1 \mathrm{~h}$ of treatment. On the other hand, treatment with PDGF and TGF $\beta$ both induced migration of the cultured HSCs but did not show significant increase on p-AKT (Fig. 6d-f). These data, together with the moderate change observed for PTEN and p-AKT expression in HSCs isolated from Pten null livers (Fig. 3a), indicate that the intrinsic signal due to PTEN loss in HSCs is an unlikely mechanism for their activation in the injured Pten null liver and that fibrosis is likely due to secondary effects, e.g., resulting from the underlying liver injury.

To test this in vivo, we induced injury in the Pten/Akt2 double null mice where injury does not occur spontaneously. Inducing injury with DDC in these mice led to robust expression of markers for fibrosis (Fig. $6 \mathrm{~g}$ ), including Colla1, Timp 1, and SMA $\alpha$. Expression of desmin and p75 NTR is also moderately induced by treatment of DDC. This observation suggests that the induction of these genes do not rely on the signals of AKT2 but do require injury.
Wnt signaling mediates HSC activation in injured steatotic Pten null liver

We showed previously that AKT2 loss inhibits liver injury induced by Pten deletion [6]. In the same study, we also showed that expression of Wnt7a and 10a and Fzd2, a Wnt receptor, is induced in the Pten null liver [6]. Wnt signal may be a potential activator of fibrosis $[12,13]$. To test the hypothesis that lipotoxic injury induces Wnt to promote fibrosis development, we first confirm that with the exception of Wnt 5a, Wnt 7a, 7b, and 10a expressions are all induced in 9-month-old Pten null steatotic livers and returned to control levels or lower in the non-steatotic Pten/Akt2 double null livers where Pten is still deleted and Akt2 loss rescued steatosis (Fig. 7a). We also found that though Wnt 5a levels did not increase, sFRP5a levels are significantly reduced (Fig. 7b).

Wnt 5a was found to inhibit the differentiation and accumulation of lipid in adipocyte and favors the elongated morphology, whereas its inhibitor soluble fizzled-related protein 5a (sFRP5a) does the opposite [14-16]. To test whether aWnt5a-sFRP5a-like signaling may play a role
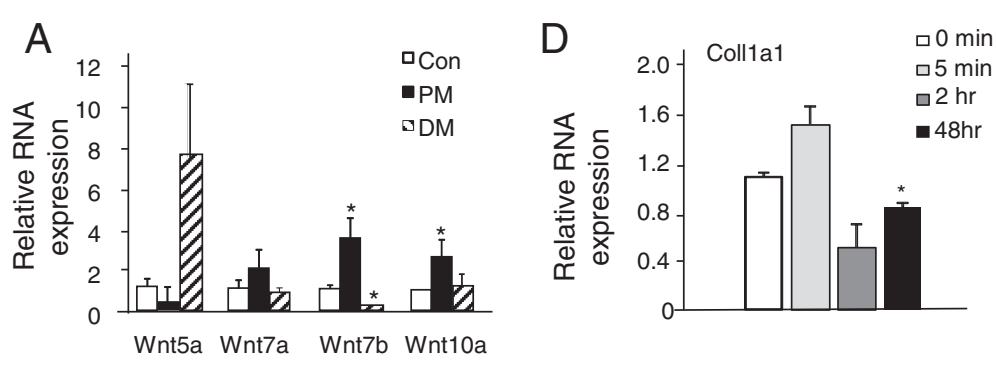

B
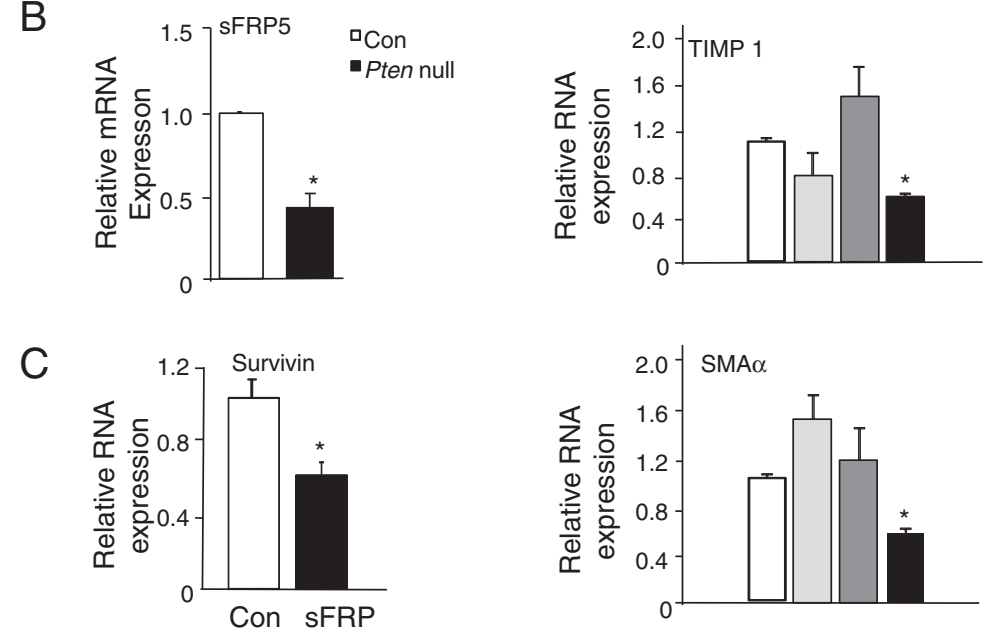

Fig. 7 Activation of $\beta$-catenin leads to the activation of HSCs. a. Expression of Wnt ligands Wnt5a, 7a, 7b, and 10a in control (Con), Pten null (Pm), and Pten/Akt2 double mutant $(\mathrm{Dm})$ livers. ${ }^{*}$ significantly different from the control groups of the same gene at $p<0.05 . n=3$. $\mathbf{b}$ Expression of Wnt 5 a alternative receptor sFRP5 in the control (Con, open bar) and Pten null liver (solid bar). *significantly different from the control groups of the same gene at $p<0.05$. $n=5$. Cultured human HSCs were treated with sFRP, and expression of fibrogenic genes was evaluated (c, d). c Survivin expression indicating that sFRP treatment reduced $\beta$-catenin transcriptional activity. $\mathbf{d}$ Expression of fibrogenic genes. ${ }^{*}$ significantly different from the control groups of the same gene at $p<0.05 . n=3$ 
in the activation of HSCs, we treated cultured rat HSCs with a human recombinant sFRP and investigated the expressions of Col1a1, SMA $\alpha$, and Timp 1 (Fig. 7c, d). While the time course response is different for each marker gene, the expression of Colla1, SMA $\alpha$, and Timp 1 all went down at 48-h posttreatment. Expression of desmin and p75NTR did not differ within this time frame (data not shown). A longer time window may be needed to observe changes in the latter two markers. In addition, treatment of HSC-Con culture with sFRP blocked the morphological switch of HSCs (Fig. 8). The HSC-Con culture remained round quiescent 6 days after the addition of sFRP, whereas the spindle-shaped activated HSC morphology progressed in the vehicle-treated cells. In the HSC-Pm culture, treatment with sFRP was ineffective (data not shown), likely because the HSCs are already activated. This data suggests that downregulation of sFRP5a and upregulation of Wnt ligands observed in the Pten null liver may have permitted the activation of HSCs in vivo.

To confirm the involvement of Wnt signal in HSC activation, we introduced siRNA against $\beta$-catenin, the target of Wnt to cultured rat HSCs (Fig. 9a). Inhibiting $\beta$ catenin activity led to reduced cell growth and migration

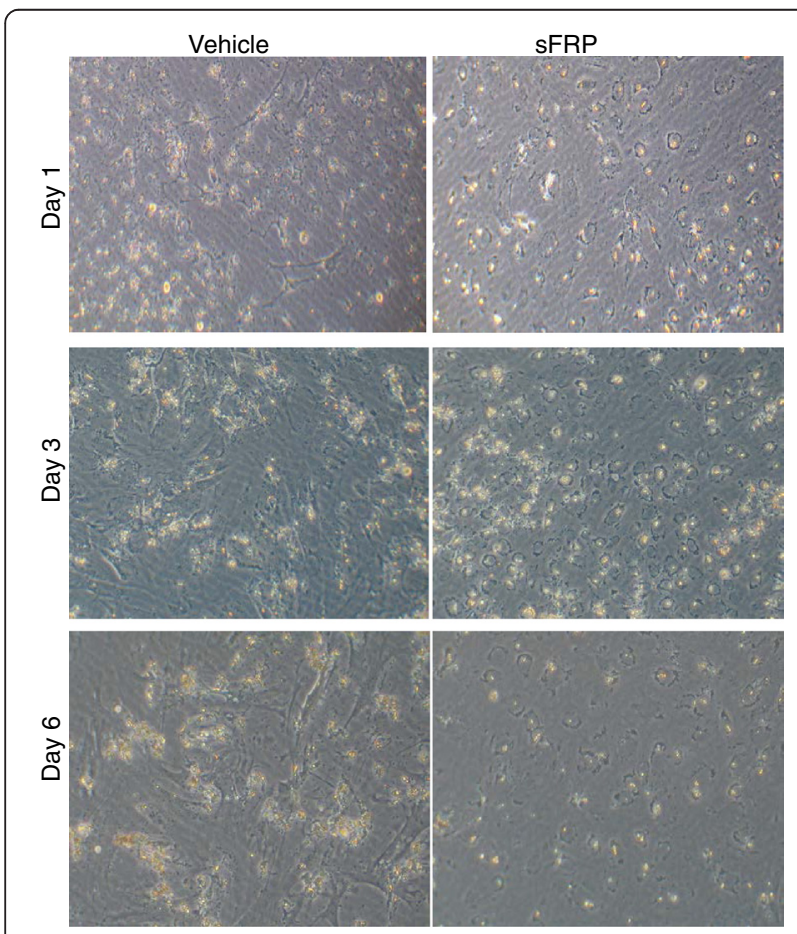

Fig. 8 Images of cultured HSCs treated with or without sFRP. HSCS isolated from control mice are treated with sFRP on the third day of culture for 1 day (day 1), 3 days (day 3), and 6 days (day 6). sFRP treatment completely blocked the activation of HSCs at D3 and D6. The morphology of HSCs in sFRP-treated cultures did not progress to spindle fiber whereas obvious spindle fiber formation is observed in the vehicle-treated cultures
(Fig. 9b, c). Particularly, significant reduction of SMAa expression (approximately threefold) was observed when $\beta$-catenin is downregulated with moderate inhibition on the expression of Colla1 and Timp 1 (Fig. 9d). We also used chemicals that are capable of interfering with $\beta$ catenin function to modulate Wnt activities in primary HSCs and determined whether activating $\beta$-catenin activity is sufficient to promote the progression of primary HSC activation and whether $\beta$-catenin activation is necessary for this process to occur. $\mathrm{LiCl}$ inhibits GSK3 $\beta$ and blocks the degradation of Wnt downstream target $\beta$-catenin [17], whereas curcumin blocks the activity of $\beta$-catenin [18] (Fig. 9e). Consistent with a positive regulatory role of $\mathrm{Wnt} / \beta$-catenin on fibrogenesis, $\mathrm{LiCl}_{3}$ treatment induced the expressions of desmin, Timp 1, and Col1a1 (Fig. 9f, left panel), whereas their expressions are moderately downregulated as a result of curcumin treatment (Fig. 9f, right panel).

Finally, we used a small molecule inhibitor of Wnt/ $\beta$ catenin, ICG-001, which specifically blocks the interaction of $\beta$-catenin with its coactivator CBP to inhibit $\beta$ catenin transcriptional activity in vivo. Fibrosis in the Pten null mice becomes obvious after 6 months of age. To advance this onset of fibrosis and induce the expression of Col 1a1, desmin, and Timp 1, markers for fibrosis, we used a cohort of 1.5-month-old mice with DDC (Fig. 9g). In these DDC-treated mice, ICG-001 was given to inhibit Wnt/CBP/ $\beta$-catenin transcription $[19,20]$. With the exception of Timp 1, expression of all fibrogenic markers are downregulated with ICG-001 to different extent including SMA $\alpha$ and p75 NTR, of which the expression did no increase with DDC treatment. Expression of Col1a1, particularly, is significantly reduced whereas the change in other makers did not reach significance. While not all markers responded in a synchronized manner to $\mathrm{LiCl}$, curcumin, si- $\beta$-catenin, or ICG001, our data is consistent with a pro-fibrogenic role of Wnt in the liver. The lack of synchrony is likely due to the nature of the markers as indicators of HSC activation rather than drivers of fibrosis. Depending on the stage of activation/differentiation of HSCs, different markers are expressed, leading to the varied response to Wnt signal-induced expression changes.

\section{Discussion}

Hepatic fibrosis is a pathological condition that follows chronic liver disease, including NASH and ASH, and is a precursor to liver cancer development. In this study, we explored the molecular link between NASH and fibrosis. We demonstrated that Pten null model is a relevant model for studying fibrosis development because (1) liver disease progresses similarly in humans and Pten null mice, (2) the Pten null mice develop progressive and severe fibrosis from 6 months on, and (3) HSCs 
A

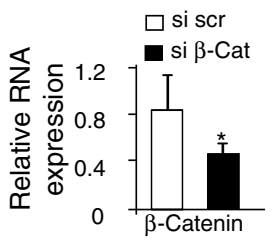

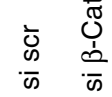

$\approx \beta-C a t$
B

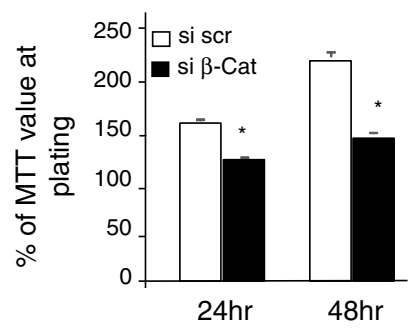

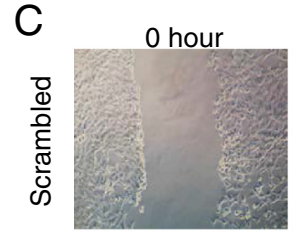
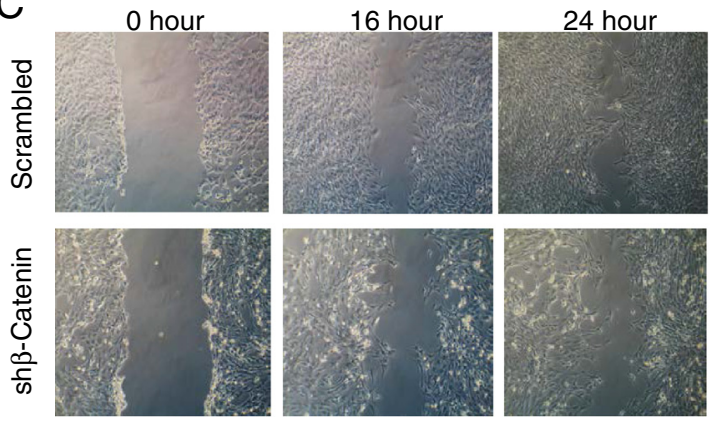

$\mathrm{E}$

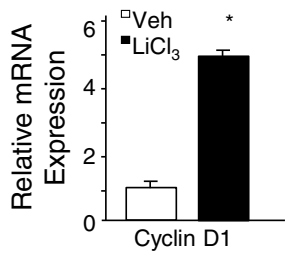

$\mathrm{F}$

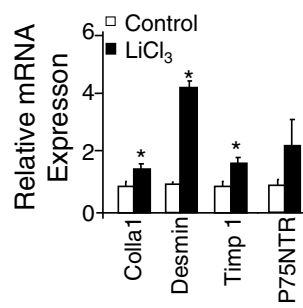

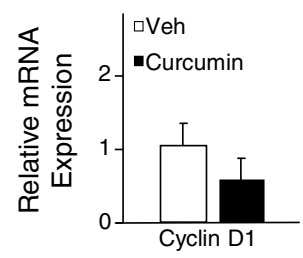

G

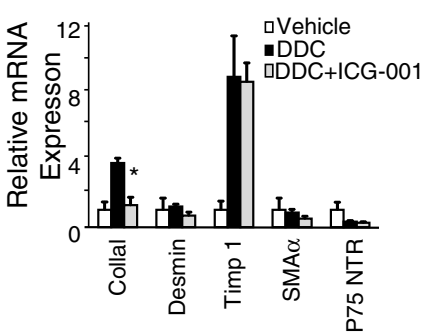

Fig. 9 Inhibition of Wnt signal blocks the activation of HSCs. a Downregulation of $\beta$-catenin with siRNA against $\beta$-catenin led to reduced expression of fibrogenic genes. Left, $\beta$-catenin mRNA expression in si-scrambled and si- $\beta$-catenin transfected HSCs. Right, protein levels of $\beta$-catenin in si-scrambled and si- $\beta$-catenin transfected HSCs. * ${ }^{*}$ ignificantly different from the control groups of the same gene at $p<0.05 . n=3$ with experiments repeated three times. b The effects of $\beta$-catenin knockdown on cell proliferation measured by a MTT assay. $n=3,{ }^{*} p<0.05$. $\mathbf{c}$ Representative images of wound healing assay in sh $\beta$-catenin knockdown cells vs. controls. $\mathbf{d}$ mRNA expression of marker genes of fibrosis in si-scrambled and si- $\beta$-catenin transfected HSCs. e LiCl activator of $\beta$-catenin, and curcumin, an inhibitor of $\beta$-catenin activity, are used to treat primary HSCS at day 2 of culture. Left panel, LiCl $\mathrm{Lreatment}_{3}$ led to the activation of $\beta$-catenin transcriptional activity indicated by an induction of cyclin D1 expression. Middle panel, curcumin treatment led to attenuated cyclin D1 expression, indicating the inhibition of $\beta$-catenin transcriptional activity. Right panel, p-GSK3 $\beta$ levels indicate that $\mathrm{LiCl}_{3}$ treatment led to the phosphorylation of GSK3 $\beta$. $\mathbf{f}$ Marker analysis indicates that $\mathrm{LiCl}_{3}$ treatment (left panel) induced expression of fibrotic markers whereas curcumin treatment (right panel) suppressed them. Open bars, vehicle-treated (control); solid bars, either $\mathrm{LiCl}_{3}$ - or Curcumin-treated cultures. ${ }^{*}$ significantly different from the control groups of the same gene at $p<0.05 . n=3$. $\mathbf{g}$ Using a $\beta$-catenin inhibitor ICG-001 to block the transcriptional activity of $\beta$-catenin reduces the expression of markers for fibrosis except Timp 1. Black bar, DDC-treated Pten null mice; gray bar, Pten mull mice treated with DDC+ICG-001; open bar, vehicle-treated controls. ${ }^{*}$ significantly different from the control groups of the same gene at $p<0.05 . n=3$

implicated in fibrosis development in humans are also activated in Pten null mice. Enhanced expression of Colla1, Timp 1, SMA $\alpha$, and p75NTR suggests the involvement of HSCs. HSCs isolated from Pten null liver are fully activated with spindle morphology and low vitamin A autofluorescence, confirming the severe fibrosis phenotype that was observed in vivo.

After demonstrating the relevance of the Pten null mice model, we utilized these mice to investigate the signaling pathways that drive fibrosis development. We found that 
(1) activation of HSC is associated with steatosis and injury but not directly caused by alterations of PTEN/ PI3K/AKT signaling, (2) blocking Wnt signaling significantly attenuated the ability of the quiescent HSCs to become activated, and (3) activation of Wnt signaling induces genes associated with activated HSCs. Taken together, (1), (2), and (3) suggest that Wnt activation resulting from steatosis induced liver injury activates HSCs and thus induces hepatic fibrosis.

In humans, fibrosis occurs in patients with viral infections, ASH, and NASH, as well as biliary and other diseases that lead to injury of liver parenchymals [21]. In animal experiments, fibrosis development is typically induced by inducing injury to liver parenchymals [1]. Injuries are typically induced using physical approaches such as ductal ligation and chemical approaches such as carbon tetrachloride treatment, choline deficient diet, alcoholic feedings, or ligation of the bile duct to cause acid buildup to damage the liver parenchymal [22, 23]. A similarity among these models and also a significant clinical interest is damage to hepatocytes and attenuation of their proliferation. In this study, rather than directly injuring the animal subjects using physical or chemical means, we used a NASH model where fat accumulation in the liver is a consequence of hyperactivated insulin signal, mimicking human NASH conditions [6]. Liver injury in the Pten null mice results from fatty liver disease much as obesity-induced fatty liver often leads to NASH in humans. We show here that NASH is accompanied by deposition of collagen fibers and activation of HSCs, and preventing NASH resolves these conditions. This study, thus, provides experimental proof that NASH does lead to fibrosis development.

AKT, the downstream kinase of PTEN, has been shown to control multiple fibrogenic genes in various tissue/cell types. In mouse skin, loss of PTEN induces the expression of fibrogenic genes [24]. Sustained expression of wild type PTEN in cultured rat HSC inhibited morphological changes associated with HSC activation [25]. Chronic activation of PI3K also induced accumulation of collagens $[10,11]$. Thus, we were surprised to find that PTEN and AKT2 signals did not change significantly in HSCs isolated from the Pten null livers. (Pten deletion is liver-cell specific in Pten null mice. Pten is not deleted in HSCs.) Our observations suggest that the effect of AKT2 on fibrogenesis is likely secondary to its role in steatosis and the subsequent liver injury.

Wnt signaling has recently been reported to be important for HSC activation [13, 26]. Liver HSCs express a number of different receptors for Wnt [27]. During culturing and activation of HSCs, the expression of Wnt receptors and ligands is induced [12]. The Wnt antagonist DKK has been found to enhance the transcriptional activity of an adipogenic gene including peroxisomal proliferation activated receptor (PPAR $\gamma$ ) [26]. Upregulation of PPAR $\gamma$ maintains the lipid droplets and allows the HSCs to remain quiescent with many lipid droplets present in each cell whereas Wnt blocks DKK signal, allowing activation of HSCs $[14,26]$. Our study shows that this signal is likely relevant to the steatosis-induced fibrosis as several Wnt ligands and receptors are induced and sFRP5 is inhibited in the injured Pten null liver [6]. Whether Wnt activation is a direct consequence of steatosis or requires injury to occur remains to be determined.

\section{Conclusions}

In summary, our data established that the liver Pten null model displays progressive fibrosis prior to cancer development. The fibrosis is a result of steatosis induced by PTEN loss, suggesting that the liver-specific Pten null mouse is a relevant model for studying the progression of liver cancer co-developed with fatty liver injuryinduced fibrosis. Our data further indicates that the Wnt signal pathway likely mediates steatosis-induced fibrosis.

\section{Methods \\ Animals}

Targeted deletion of Pten (Pten null) and Pten/Akt2 double mutant mice $(\mathrm{Dm})$ were reported previously $[5,6]$. Control (Con) animals are Pten ${ }^{\text {loxP/loxP }} ; \mathrm{Alb}-\mathrm{Cre}^{-}$. Experiments were conducted according to IACUC guidelines of the University of Southern California. Fasting glucose were measured in overnight $(16 \mathrm{~h})$ fasted mice. 3,5-dietoxycarbonyl-1,4 dihydrocollidine (DDC, $0.1 \% w / w$ diet) treatment was performed in 3-month-old mice for 5 weeks. Male animals of C57BL/6 and J129svj background from the same breeding colony were used for all experiments. For 1.5-month-old mice used for ICG-001 study, DDC $(0.05 \% w / w$ diet $)$ were given in three doses using a 2-day off 1 day on protocol. ICG-001 (5 mg/kg per day in saline) is delivered using a mini-osmotic pump that dispenses at $1 \mu \mathrm{L} / \mathrm{h}$ delivery rate. All experiments were conducted according to IACUC guidelines of the University of Southern California (Protocol \#11162).

\section{Human liver samples}

Human liver samples biopsied from fibrosis patients were obtained from Pennsylvania State University. All patient information was removed, and all experiments were conducted according to IRB guidelines of Pennsylvania State University.

\section{Database mining}

Gene expression data base GSE37031 was downloaded from NCBI website. All patient information were reported in the original publication for the database [8]. The data set was then analyzed for expressions of 
PTEN in NASH $(n=8)$ vs. non-NASH $(n=7)$ patients. Immunohistochemical staining images were obtained from the Human Protein Atlas website (http://www.proteinatlas.org/).

\section{Cell lines}

Rat HSCs [28] were cultured in $1 \mathrm{~g} / \mathrm{L}$ glucose Dulbecco's modified Eagle's medium (DMEM) supplemented with $10 \%$ fetal bovine serum (FBS), penicillin (50 units $/ \mathrm{ml}$ ), and streptomycin $(50 \mu \mathrm{g} / \mathrm{ml})$ at a $37{ }^{\circ} \mathrm{C}, 5 \% \mathrm{CO}_{2}$ incubator. For treatment with $0.1 \mu \mathrm{g} / \mathrm{ml} \mathrm{mIGF,} 50 \mathrm{ng} / \mathrm{ml}$ PDGF, or $4 \mathrm{ng} /$ $\mathrm{ml}$ TGF $\beta$, cells were serum starved for $24 \mathrm{~h}$.

\section{Primary hepatic stellate cell isolation}

HSCs were isolated using OptiPrep gradient ultracentrifugation [12]. HSC fraction was collected from the medium and 1.034 interface of OptiPrep gradients, and cultured and morphological changes of HSCs are monitored using both light and fluorescent microscopy to monitor for vitamin A autofluorescence at indicated time points. RNAs were isolated for marker analysis to monitor the activation of HSCs. In experiment using lithium chloride $\left(\mathrm{LiCl}_{3}\right), \mathrm{LiCl}_{3}(10 \mathrm{mM})$ was added to the day 1 culture and incubated for 2 days before the collection of RNA. In experiments using curcumin, 10$\mu \mathrm{M}$ curcumin was added to day 1 culture and RNAs were collected 2 days after the addition of curcumin. Frizzled-related protein 1 (sFRP1) (R\&D systems, Minneapolis, MN) is used at $40 \mathrm{ng} / \mathrm{ml}$ added to day $3 \mathrm{HSC}$ culture and morphology followed for 7 days.

\section{Immunohistochemistry}

Liver sections were stained with hematoxylin and eosin (H\&E) for morphology and Sirius Red to visualize fibrotic fibers. Sirius Red staining is quantified using Image J. Three animals per groups were quantified, and three randomly chosen areas per animal were assessed. Anti-smooth muscle actin $\alpha$ (SMA $\alpha$ ) (Sigma-Aldrich) was used in indirect immunohistochemistry to further confirm the buildup of extracellular matrix.

\section{Quantitative PCR}

Total RNA $(2 \mu \mathrm{g})$ from liver tissues was used for qPCR analysis for gene expression. Primers used are SMA $\alpha$, desmin, Colla1, and p75NTR as previously indicated [29]. Primers for Timp 1 are: 5'-CAGTAAGGCCTGTAGCTGT GC 5'CTCGTTGATTTCGGGGAAC. GAPDH were detected for internal controls.

\section{Protein electrophoresis}

Protein lysates $(40 \mu \mathrm{g})$ were loaded from each sample for electrophoresis using polyacrylamide gels. Membranes were probed with antibodies for PTEN, p-AKT, p-GSK3 $\beta$, and GSK3 $\beta$ (cell signaling). $\alpha$-Actin (Sigma) protein expression is used as loading controls.

\section{Statistics}

Data were subjected to Student's $t$ tests for two sample comparisons. In cases of more than two groups, multivariate ANOVA was used to determine the statistical differences followed by pairwise comparison using Fischer's LSD test. $p \leq 0.05$ is considered to be statistically significant. Data are presented as mean \pm SEM.

\begin{abstract}
Acknowledgements
This work was supported by R01CA154986-01, a pilot grant from the USC Liver Center program grant P30DK48522 as well as funding from Zumberg foundation (BLS). BLS also acknowledges NIDDK R21 DK075928-02 and R01DK084241-01. We would also acknowledge funding from NIH grants R01-AA020753 (to KA) and R24-AA12885 Non-parenchymal Liver Cell Core (to KA and JW). We also acknowledge support from USC Norris Comprehensive Cancer Center Support Grant P30 CA014089. NIH 1R01CA166161-01A1 and NIH 1R01 HL112638-01 are gratefully acknowledged (MK). We thank Dr. Bryan Stiles for his help with editing this manuscript.
\end{abstract}

\section{Authors' contributions}

$\mathrm{LH}, \mathrm{GJ}$, and BLS study concept and design and drafting of the manuscript. VM, JW and FF acquisition of data. ZP, KA, BLS and CBR analysis and interpretation of data; statistical analysis. MK provide key reagents. KA, CBR and BLS critical revision of the manuscript for important intellectual content. All authors read and approved the final manuscript.

\section{Competing interests}

The authors declare that they have no competing interests.

\section{Ethics approval and consent to participate}

All experiments were conducted according to IACUC guidelines of the University of Southern California (Protocol \#11162). All patient information was removed, and all experiments were conducted according to IRB guidelines of Pennsylvania State University.

\section{Author details}

${ }^{1}$ Pharmacology and Pharmaceutical Sciences, School of Pharmacy, University of Southern California, Los Angeles, CA 90033, USA. ${ }^{2}$ Imperial College, London, England. ${ }^{3}$ Pathology, Keck School of Medicine, University of Southern California, Los Angeles, CA 90033, USA. ${ }^{4}$ Department of Biochemistry and Molecular Biology, Keck School of Medicine, University of Southern California, Los Angeles, CA 90033, USA. ${ }^{5}$ Department of Pediatrics and Pharmacology, Pennsylvania State University College of Medicine, 500 University Drive, H085, Hershey, PA 17033, USA. ${ }^{\text {P }}$ harmacology and Pharmaceutical Sciences, USC School of Pharmacy PSC402, 1985 Zonal Ave, Los Angeles, CA 90089, USA.

Received: 19 January 2016 Accepted: 31 May 2016

Published online: 14 June 2016

\section{References}

1. Weber SN, Lammert F. Genetics of liver injury and fibrosis. Alcohol Clin Exp Res. 2011;35:800-3.

2. Brenner DA. Molecular pathogenesis of liver fibrosis. Trans Am Clin Climatol Assoc. 2009;120:361-8.

3. Sato M, Suzuki S, Senoo H. Hepatic stellate cells: unique characteristics in cell biology and phenotype. Cell Struct Funct. 2003;28:105-12.

4. Hernandez-Gea V, Friedman SL. Pathogenesis of liver fibrosis. Annu Rev Pathol. 2011;6:425-56.

5. Stiles B, Wang Y, Stahl A, Bassilian S, Lee WP, Kim YJ, et al. Liver-specific deletion of negative regulator Pten results in fatty liver and insulin hypersensitivity [corrected]. Proc Natl Acad Sci U S A. 2004;101:2082-7.

6. Galicia VA, He L, Dang H, Kanel G, Vendryes C, French BA, et al. Expansion of hepatic tumor progenitor cells in Pten-null mice requires liver injury and is reversed by loss of AKT2. Gastroenterology. 2010;139:2170-82. 
7. He L, Hou X, Kanel G, Zeng N, Galicia V, Wang Y, et al. The critical role of AKT2 in hepatic steatosis induced by PTEN loss. Am J Pathol. 2010;176:2302-8.

8. Lopez-Vicario C, Gonzalez-Periz A, Rius B, Moran-Salvador E, Garcia-Alonso V, Lozano JJ, et al. Molecular interplay between Delta5/Delta6 desaturases and long-chain fatty acids in the pathogenesis of non-alcoholic steatohepatitis. Gut. 2014;63:344-55.

9. Mohammed FF, Pennington CJ, Kassiri Z, Rubin JS, Soloway PD, Ruther U, et al. Metalloproteinase inhibitor TIMP-1 affects hepatocyte cell cycle via HGF activation in murine liver regeneration. Hepatology. 2005;41:857-67.

10. Reif S, Lang A, Lindquist JN, Yata Y, Gabele E, Scanga A, et al. The role of focal adhesion kinasephosphatidylinositol 3-kinase-akt signaling in hepatic stellate cell proliferation and type I collagen expression. J Biol Chem. 2003; 278:8083-90.

11. Son G, Hines IN, Lindquist J, Schrum LW, Rippe RA. Inhibition of phosphatidylinositol 3-kinase signaling in hepatic stellate cells blocks the progression of hepatic fibrosis. Hepatology. 2009;50:1512-23.

12. Cheng JH, She H, Han YP, Wang J, Xiong S, Asahina K, et al. Wnt antagonism inhibits hepatic stellate cell activation and liver fibrosis. Am J Physiol Gastrointest Liver Physiol. 2008;294:G39-49.

13. Myung SJ, Yoon JH, Gwak GY, Kim W, Lee JH, Kim KM, et al. Wnt signaling enhances the activation and survival of human hepatic stellate cells. FEBS Lett. 2007:581:2954-8.

14. Christodoulides C, Lagathu C, Sethi JK, Vidal-Puig A. Adipogenesis and WNT signalling. Trends Endocrinol Metab. 2009;20:16-24.

15. Park JR, Jung JW, Lee YS, Kang KS. The roles of Wnt antagonists Dkk1 and SFRP4 during adipogenesis of human adipose tissue-derived mesenchymal stem cells. Cell Prolif. 2008:41:859-74.

16. Taipaleenmaki H, Abdallah BM, AIDahmash A, Saamanen AM, Kassem M. Wnt signalling mediates the cross-talk between bone marrow derived pre-adipocytic and pre-osteoblastic cell populations. Exp Cell Res. 2011;317:745-56.

17. Stambolic V, Ruel L, Woodgett JR. Lithium inhibits glycogen synthase kinase-3 activity and mimics wingless signalling in intact cells. Curr Biol. 1996:6:1664-8

18. Jaiswal AS, Marlow BP, Gupta N, Narayan S. Beta-catenin-mediated transactivation and cell-cell adhesion pathways are important in curcumin (diferuylmethane)-induced growth arrest and apoptosis in colon cancer cells. Oncogene. 2002;21:8414-27.

19. Ma H, Nguyen C, Lee KS, Kahn M. Differential roles for the coactivators CBP and p300 on TCF/betacatenin-mediated survivin gene expression. Oncogene. 2005;24:3619-31.

20. Teo JL, Ma H, Nguyen C, Lam C, Kahn M. Specific inhibition of CBP/beta-catenin interaction rescues defects in neuronal differentiation caused by a presenilin-1 mutation. Proc Natl Acad Sci U S A. 2005;102:12171-6.

21. Pinzani M, Rosselli M, Zuckermann M. Liver cirrhosis. Best Pract Res Clin Gastroenterol. 2011;25:281-90.

22. Guyot C, Combe C, Balabaud C, Bioulac-Sage P, Desmouliere A. Fibrogenic cell fate during fibrotic tissue remodelling observed in rat and human cultured liver slices. J Hepatol. 2007:46:142-50.

23. Tsukamoto H, Towner SJ, Ciofalo LM, French SW. Ethanol-induced liver fibrosis in rats fed high fat diet. Hepatology. 1986;6:814-22.

24. Parapuram SK, Shi-wen X, Elliott C, Welch ID, Jones H, Baron M, et al. Loss of PTEN expression by dermal fibroblasts causes skin fibrosis. J Invest Dermatol. 2011;131:1996-2003.

25. Takashima M, Parsons CJ, Ikejima K, Watanabe S, White ES, Rippe RA. The tumor suppressor protein PTEN inhibits rat hepatic stellate cell activation. J Gastroenterol. 2009;44:847-55.

26. Zhu NL, Asahina K, Wang J, Ueno A, Lazaro R, Miyaoka Y, et al. Hepatic stellate cell-derived delta-like homolog 1 (DLK1) protein in liver regeneration. J Biol Chem. 2012;287:10355-67.

27. Zeng G, Awan F, Otruba W, Muller P, Apte U, Tan X, et al. Wnt'er in liver: expression of Wnt and frizzled genes in mouse. Hepatology. 2007;45:195-204.

28. Xiong S, Yavrom S, Hazra S, Wu D, She H. Spontanously immortalized cell line from rat liver fibrosis with phenotypes of both hepatic stellate cells and myofibroblasts. Hepatology. 2001;34:520A.

29. Asahina K, Tsai SY, Li P, Ishii M, Maxson Jr RE, Sucov HM, et al. Mesenchymal origin of hepatic stellate cells, submesothelial cells, and perivascular mesenchymal cells during mouse liver development. Hepatology. 2009;49: 998-1011.

\section{Submit your next manuscript to BioMed Central and we will help you at every step:}

- We accept pre-submission inquiries

- Our selector tool helps you to find the most relevant journal

- We provide round the clock customer support

- Convenient online submission

- Thorough peer review

- Inclusion in PubMed and all major indexing services

- Maximum visibility for your research

Submit your manuscript at www.biomedcentral.com/submit 